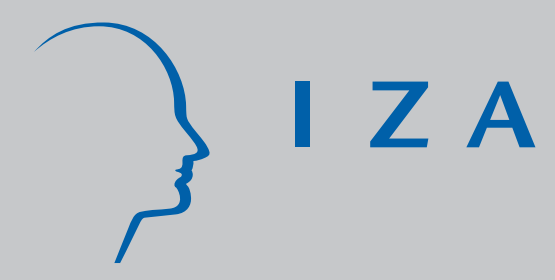

IZADP No. 3943

The Wooldridge Method for the Initial Values Problem Is Simple: What About Performance?

Alpaslan Akay

J anuary 2009 


\title{
The Wooldridge Method for the Initial Values Problem Is Simple: What About Performance?
}

\author{
Alpaslan Akay \\ IZA \\ Discussion Paper No. 3943 \\ January 2009 \\ IZA \\ P.O. Box 7240 \\ 53072 Bonn \\ Germany \\ Phone: +49-228-3894-0 \\ Fax: +49-228-3894-180 \\ E-mail: iza@iza.org
}

\begin{abstract}
Any opinions expressed here are those of the author(s) and not those of IZA. Research published in this series may include views on policy, but the institute itself takes no institutional policy positions.

The Institute for the Study of Labor (IZA) in Bonn is a local and virtual international research center and a place of communication between science, politics and business. IZA is an independent nonprofit organization supported by Deutsche Post World Net. The center is associated with the University of Bonn and offers a stimulating research environment through its international network, workshops and conferences, data service, project support, research visits and doctoral program. IZA engages in (i) original and internationally competitive research in all fields of labor economics, (ii) development of policy concepts, and (iii) dissemination of research results and concepts to the interested public.
\end{abstract}

IZA Discussion Papers often represent preliminary work and are circulated to encourage discussion. Citation of such a paper should account for its provisional character. A revised version may be available directly from the author. 


\section{ABSTRACT}

\section{The Wooldridge Method for the Initial Values Problem Is Simple: What About Performance?*}

The Wooldridge method is based on a simple and novel strategy to deal with the initial values problem in the nonlinear dynamic random-effects panel data models. This characteristic of the method makes it very attractive in empirical applications. However, its finite sample performance is not known as of yet. In this paper we investigate the performance of this method in comparison with an ideal case in which the initial values are known constants, the worst scenario based on exogenous initial values assumption, and the Heckman's reducedform approximation method which is widely used in the literature. The dynamic randomeffects probit and tobit (type1) models are used as the working examples. Various designs of Monte Carlo Experiments with balanced and unbalanced panel data sets, and also two full length empirical applications are provided. The results suggest that the Wooldridge method works very well for the panels with moderately long durations (longer than 5-8 periods). In short panels Heckman's reduced-form approximation is suggested (shorter than 5 periods). It is also found that all methods perform equally well for panels of long durations (longer than 10-15 periods).

JEL Classification: $\quad$ C23, C25

Keywords: initial values problem, dynamic probit and tobit models, Monte Carlo experiment, Heckman's reduced-form approximation, Wooldridge method

Corresponding author:

Alpaslan Akay

IZA

P.O. Box 7240

53072 Bonn

Germany

E-mail: akay@iza.org

\footnotetext{
* I thank Steven Bond, Lennart Flood, Konstantinos Tatsiramos, Arne Uhlendorff, Måns Söderbom, Roger Wahlberg, Elias Tsakas, Peter Martinsson, Melanie Khamis and seminar participants at Gothenburg University for their valuable comments.
} 


\section{Introduction}

One of the crucial issues in the estimation of a dynamic panel data random-effects models is the initial values problem (Blundell and Smith, 1991; Arellano and Bover, 1997; Blundell and Bond, 1998; Arellano and Honore, 2001; Honore, 2002; Arellano and Carrasco, 2003; De Jong and Herrera, 2005; Arellano and Hahn, 2005; Wooldridge, 2005; Honore and Tamer, 2006). This problem occurs when the history of a stochastic process is not observed from the very beginning. Almost all panel data sets used in microeconometric practice today contain information about individuals entered into the process before the observation period. Ad hoc treatments of this problem are prone to bias and inconsistent estimators as well as a wrong inference of the magnitude of true (structural) and spurious state dependence (Heckman, 1981; Honore, 2002; Hsiao, 2003; Honore and Tamer, 2006).

This problem is either ignored by assuming that the initial values have not been in effect with what happened in the unknown past, i.e. exogenous variables, independent from the exogenous variables and the unobserved individual-effects (heterogeneity), or the stochastic process underlying the model is assumed as is in the steady state (Heckman, 1981; Card and Sullivan, 1988). The exogenous initial values assumption is very naive and may lead to severe bias if the initial observations have been created with the evolution of observed and unobserved characteristics in the past. The initial stationary assumption is also very unattractive especially when age-trended variables drive the process (Heckman, 1981, Hsiao, 2003).

A realistic solution strategy is first suggested by Heckman (1981) which considers the initial values are endogenous variables with a probability distribution condi- 
tioned on the exogenous variables and unobserved individual-effects. The strategy of the method is to approximate the conditional probability of initial values with reduced-form equations using available pre-sample information. This leads to very flexible functional forms. Using a small scale Monte Carlo study, Heckman (1981) shows that this solution method performs very well. The main problem of this method in practice is that the approximation of the conditional probability of initial values leads to a simultaneous estimation problem of the reduced-form and structural model which can create a large computational burden in the estimation process.

One other way to ensure that initial values are not a problem in the estimation is to use a fixed-effects approach. The conditional distribution of unobserved individual-effects does not play a role in the estimation process of this approach. However, the fixed-effects approach can be seriously biased as it suffers from socalled incidental parameters problem (Neyman and Scott, 1948). Alternatively, some of the recently developed nonparametric methods can be used (Honore and Kyriazidou, 2000; Hu, 2002; Honore, 2003). For instance, Honore and Kyriazidou (2000) provide a fixed-effects logit model based on kernel-weighted GMM estimators which also absorb the initial values problem. These estimators though have some problems. For instance, it is not possible to calculate average partial effects (Wooldridge, 2005). Thus, the random-effects specification is still attractive in practice and a solution for the initial values problem is inevitable.

Wooldridge (2005) has recently provided a very simple alternative to the Heckman's reduced-form approximation to solve the initial values problem. This method leads to very simple and tractable likelihoods that is not different than standard sta- 
tic random-effects model. It is based on an auxiliary distribution of the unobserved individual-effects which is conditioned on the initial values and exogenous variables. It is also very useful in random-effects specifications as it is very similar to Chamberlain approach (1984) with which one can also deal with the possible correlation between exogenous variables and unobserved individual-effects. To our knowledge, although there is a growing literature which routinely uses this method in empirical applications (Contayannis et al., 2004), there is no rigorous study on the finite sample performance of the method as an alternative to Heckman's reduced-form approximation.

The main aim of this paper is to provide Monte Carlo Experiments $(M C E)$ and present real data evidence to investigate the finite sample performance of the Wooldridge method. The dynamic panel data random-effects probit and tobit (type I) models are used as working examples. The paper also aims to compare the performance of the Wooldridge method with the results produced by the exogenous initial values assumption and the Heckman's reduced-form approximation. First, we conduct various designs of $M C E$ with balanced panel data sets. However, as explained in Honore (2002), ad hoc treatments of the initial values problem are especially unappealing with unbalanced panel data sets. This is generally the case in the empirical applications and thus, various designs of $M C E$ with unbalanced panel data sets are also presented. The real microeconomic panel data sets contain more complicated structure compared to any $M C E$ design. The paper also gives two full scale real data applications which concentrate on intertemporal labor force participation and hours of work decisions of married women in Sweden between 1992 
and 2001.

Given that the aim of estimating a dynamic panel data models is to identify the different sources of persistence, the initial values problem plays a very important role. The result suggests that misspecification of the conditional distribution of the initial values leads to a serious bias on both persistence which is due to structural or spurious reasons. The exogenous initial values assumption, for instance, would lead to overestimation of the true and underestimation of the spurious state dependence. We find that the Wooldridge method works very well but it is not as successful as Heckman's reduced-form approximation for very short panels (shorter than 5). The Wooldridge method gives almost the same results with the Heckman's method for moderately long panels (longer than $5-8$ periods). One of the other and very intuitive result is that the performance of all methods tends to be equal for the panels of very long durations (longer than $10-15$ periods).

The paper is organized as follows: the next section will summarize the models and estimation strategies with different solution methods of the initial values problem. Section 3 presents our $M C E$ designs and the results. Section 4 gives two empirical applications to the intertemporal labour force participation and hours of work decisions of married women in Sweden between 1992 and 2001. Section 5 concludes. 


\section{The dynamic probit and tobit models and the}

\section{solution strategies for the initial values problem}

The process with one lag of observed dependent variable that generate the outcome is as follows. ${ }^{1}$

$$
\begin{gathered}
y_{i t}^{*}=x_{i t}^{\prime} \beta+\gamma y_{i, t-1}+\epsilon_{i t} \\
y_{i 1}^{*}=x_{i 1}^{\prime} \beta+\epsilon_{i 1}
\end{gathered}
$$

where the dependent variable $y_{i t}^{*}$ is latent (unobserved) and $y_{i 1}^{*}$ are the initial values of the process; $\epsilon_{i t}=\alpha_{i}+u_{i t}$ are the composite error terms, and $i=1, \ldots, N$, $t=2, \ldots, T ; x_{i t}$ is a vector of strictly exogenous variables in a sense that they are independent from all past, current and the future values of the disturbance $u_{i t}$; $\alpha_{i}$ is the time-persistent unobserved individual-effects which is assumed as $\alpha_{i} \sim$ $i i d N\left(0, \sigma_{\alpha}^{2}\right)$. It is also assumed to be orthogonal to exogenous variables following the standard random-effects assumption.

The dependent variable is latent and it can be observed with a criteria that determines the type of the model. The system (1-2) is a dynamic random-effect probit model if $y_{i t}=\mathbf{1}\left(y_{i t}^{*}>0\right)$ and $y_{i 1}=\mathbf{1}\left(y_{i 1}^{*}>0\right)$, where $\mathbf{1}($.$) is the indicator$ function. The error terms of the probit model are assumed as $u_{i t} \sim \operatorname{iidN}\left(0, \sigma_{u}^{2}\right)$ and $\sigma_{u}^{2}=1$, due to identification. The correlation between two sequential error terms is $\operatorname{Corr}\left(\epsilon_{i t}, \epsilon_{i k}\right)=\sigma_{\alpha}^{2} /\left(\sigma_{\alpha}^{2}+1\right),(t, k=1, \ldots, T ; t \neq k)$. The system (1-2) is a

The other alternative is to consider that the lagged values of the dependent variable is also latent (unobserved). Considering the lagged dependent variable as observed or latent leads to different implications in both economic and estimation terms. See Honore (1993), Hu (2002) and Hsiao (2003) for useful discussions. 
dynamic random-effects tobit (type I) model if the criteria is $y_{i t}=\max \left(0, y_{i t}^{*}\right)$ and $y_{i 1}=\max \left(0, y_{i 1}^{*}\right)$, where the error terms are assumed as $u_{i t} \sim \operatorname{iidN}\left(0, \sigma_{u}^{2}\right)$. The likelihood for the probit (pro) and tobit (tob) models at time $t(t>1)$ for any individual $i$ is given by,

$$
\begin{aligned}
& f_{i t}^{\text {pro }}\left(y_{i t} \mid y_{i, t-1}, x_{i t}, \alpha_{i} ; \theta\right)=\Phi\left\{D_{i t}\left(x_{i t}^{\prime} \beta+\gamma y_{i, t-1}+\sigma_{\alpha} \alpha_{i}\right)\right\} \\
& f_{i t}^{t o b}\left(y_{i t} \mid y_{i, t-1}, x_{i t}, \alpha_{i} ; \theta\right)=\left\{\begin{array}{ll}
1-\Phi\left[\left(x_{i t}^{\prime} \beta+\gamma y_{i, t-1}+\sigma_{\alpha} \alpha_{i}\right) / \sigma_{u}\right] & , y_{i t}=0 \\
\left(1 / \sigma_{u}\right) \phi\left[\left(y_{i t}-x_{i t}^{\prime} \beta-\gamma y_{i, t-1}-\sigma_{\alpha} \alpha_{i}\right) / \sigma_{u}\right], & y_{i t}>0
\end{array}\right\}
\end{aligned}
$$

where $D_{i t}=\left(2 y_{i t}-1\right), \Phi$ denotes the distribution function, $\phi$ denotes the density function of the standard normal random variable, and $\theta^{\text {pro }}=\left[\begin{array}{lll}\beta & \gamma & \sigma_{\alpha}\end{array}\right]^{\prime}, \theta^{t o b}=$ $\left[\begin{array}{llll}\beta & \gamma & \sigma_{\alpha} & \sigma_{u}\end{array}\right]^{\prime}$ are the parameter vectors to be estimated.

Given the probability distribution of the initial values which is conditioned on the exogenous variables and unobserved individual-effects $f_{1}^{\text {pro }}\left(y_{i 1} \mid\left\{x_{i t}\right\}_{t=1}^{T}, \alpha_{i} ; \theta\right)$ and $f_{1}^{t o b}\left(y_{i 1} \mid\left\{x_{i t}\right\}_{t=1}^{T}, \alpha_{i} ; \theta\right)=\left\{f_{1\left[y_{i 1}=0\right]}, f_{1\left[y_{i 1}>0\right]}\right\}$, and the exogenous variables $x_{i t}$, the general form of the full log-likelihood function for both models can be specified as follows:

$$
\log \mathcal{L}=\sum_{i=1}^{N} \ln \left[\int_{-\infty}^{\infty}\left[f_{1}\left(y_{i 1} \mid\left\{x_{i t}\right\}_{t=1}^{T}, \alpha_{i} ; \theta\right) \prod_{t=2}^{T} f_{i t}\left(y_{i t} \mid y_{i, t-1}, x_{i t}, \alpha_{i} ; \theta\right)\right] f\left(\alpha_{i}\right) d \alpha_{i}\right]
$$

Maximization of the likelihood function (5) is straightforward for both specifications, if the conditional distribution of the initial values is known. Obviously, the ideal case would be that the observed panel data set starts just together with the stochastic process, and the initial values $y_{i 1}$ are known constants (nonstochas- 
tic). In this case, there is no need to deal with the initial values problem and there is no reason to specify a conditional distribution (Heckman, 1981; Honore, 2002; Hsiao, 2003). However, if the observed sample data are observed after the process is operated many periods, the initial values would not be known and a conditional distribution for the initial values must be specified. If there is no access to $S$ periods before the sample panel data are observed $(t=S+1, S+2, \ldots, S+T)$, the conditional distribution of the initial values, $y_{i, S+1}$, is specified as $f_{i, S+1}\left(y_{i, S+1} \mid\left\{x_{i t}\right\}_{t=1}^{S+T}, \alpha_{i} ; \theta\right) .{ }^{2}$ In empirical applications, it is sometimes naively assumed that initial values have not been in effect with the unobserved $S$ periods. This assumption implies that the initial values are exogenous variables, independent from exogenous variables and unobserved individual-effects. In this case, the conditional distribution of the initial values would be equal to the marginal distribution $f_{i 1}\left(y_{i 1}\right)$ and it can be taken outside the maximization procedure of the likelihood function (5). Therefore, assuming exogenous initial values actually means ignoring the problem. If the data is not collected at the beginning of the process, and if the disturbances that generate the process are serially correlated (which is inevitable in the presence of the unobserved individual-effects), then the exogenous initial values assumption is too strong and causes serious consequences such as bias and inconsistency in the estimators (Heckman, 1981; Hyslop, 1999; Honore, 2002). ${ }^{3}$

The more realistic approach is to assume that the initial values are endogenous,

2 For notational simplicity, we will later drop the notation $S+t$ and simply use $f_{i 1}\left(y_{i 1} \mid\left\{x_{i t}\right\}_{t=1}^{T}, \alpha_{i} ; \theta\right)$. We assume that $S$ periods is passed and thus here $t=1$ (the first period of the panel data set) means $S+1$.

3 Note that, in this paper, we assume that the actual disturbance process is serially uncorrelated (such as first order autocorrelation $\mathrm{AR}(1)$ ) and the dynamic feature of the model is obtained by including a lagged dependent variable. 
and to specify a conditional distribution. However, it is not an easy to find a closed-form expression for the conditional distribution. One possibility is to assume that the conditional distribution of initial values is to be at the steady state (in equilibrium). This assumption is useful in some cases, but it is still difficult to find a closed-form expression for the conditional distribution even for the simplest case where there are no explanatory variables (Card and Sullivan, 1988). Additionally the initial stationarity assumption is also very strong if some age-trended variables are driving the process (Heckman, 1981; Hyslop, 1999; Hsiao, 2003).

Heckman (1981) suggests an approximation method for the conditional distribution of the initial values using a reduced-form equation which is based on available pre-sample information. Consider the following reduced-form equations,

$$
\begin{aligned}
& y_{i 1}^{*}=z_{i 1}^{\prime} \pi+\epsilon_{i 1} \\
& \epsilon_{i 1}=\lambda \alpha_{i}+u_{i 1}
\end{aligned}
$$

where $z_{i 1}$ is the vector of available strictly exogenous instruments which will be used as pre-sample information. This vector can consist of the first observations of the exogenous explanatory variables which are available in the sample panel data set. $\pi$ and $\lambda$ are the nuisance parameters to be estimated. $\epsilon_{i 1}$ is correlated with $\alpha_{i}$ but it is uncorrelated with $u_{i t}$ for $t>1$. The reduced-form can be expressed for probit model as $y_{i 1}=\mathbf{1}\left(y_{i 1}^{*}>0\right)$ and for tobit model as $y_{i 1}=\max \left(0, y_{i 1}^{*}\right)$. The conditional 
distribution of the initial values then can be approximated as

$$
\begin{aligned}
& f_{i 1}^{\text {pro }}\left(y_{i 1} \mid z_{i 1}, \alpha_{i} ; \pi, \lambda\right)=\Phi\left\{D_{i 1}\left(z_{i 1} \pi+\lambda \sigma_{\alpha} \alpha_{i}\right)\right\} \\
& f_{i 1}^{t o b}\left(y_{i 1} \mid z_{i 1}, \alpha_{i} ; \pi, \lambda\right)=\left\{\begin{array}{lc}
1-\Phi\left[\left(z_{i 1}^{\prime} \pi+\lambda \sigma_{\alpha} \alpha_{i}\right) / \sigma_{u}\right] & , \quad y_{i 1}=0 \\
\left(1 / \sigma_{u}\right) \phi\left[\left(y_{i 1}-z_{i 1}^{\prime} \pi-\lambda \sigma_{\alpha} \alpha_{i}\right) / \sigma_{u}\right], & y_{i 1}>0
\end{array}\right\}
\end{aligned}
$$

Simultaneous estimation of the parameters of the structural equation (1) and reducedform in (6-7) can be obtained by simply substituting (8) or (9) into the log-likelihood function (5) without imposing any restrictions (Heckman, 1981; Hsiao, 2003).

Wooldridge (2005) suggests a simple alternative to Heckman's reduced-form approximation, which is based on unobserved individual-effects as conditional on the initial values and exogenous variables. Specifying the distribution of unobserved individual-effects on these variables can lead to very tractable functional forms and consistent estimators not only for dynamic panel data random-effects probit or tobit models but also for many other dynamic nonlinear panel data models such as logit, poisson or sample selection (tobit type II).

The Wooldridge method suggests specifying $f\left(\alpha_{i} \mid\left\{x_{i t}\right\}_{t=1}^{T}, y_{i 1}\right)$ instead of $f_{i 1}($. as a similar strategy to Chamberlain (1984) correlated random-effects model. Simply, this method uses the following auxiliary distribution of the unobserved individualeffects which is conditioned on the initial values, $y_{i 1}$, and the within-means of timevariant explanatory variables, $\bar{x}_{i}$ :

$$
\alpha_{i}=\xi_{0}+\xi_{1} y_{i 1}+\xi_{2} \bar{x}_{i}+\eta_{i}
$$

where $\eta_{i}$ is a new unobserved individual-effects which is simply assumed as $\eta_{i} \sim$ 
$i i d N\left[0, \sigma_{\eta}^{2}\right] ; \alpha_{i} \mid \bar{x}_{i}, y_{i 1} \sim N\left[\xi_{0}+\xi_{1} y_{i 1}+\xi_{2} \bar{x}_{i}, \sigma_{\eta}^{2}\right] ;$ and $\bar{x}_{i}=\frac{1}{T} \sum_{t=1}^{T} x_{i t} \cdot{ }^{4}$ Thus, we obtain a conditional likelihood which is based on the joint distribution of observations conditional on initial values. The resulting likelihood function will be like those in standard static random-effect probit and tobit models. The parameters can be easily estimated by using a standard random-effects command in available softwares.

The likelihood functions of probit and tobit models (5) involve only a single integral, which can be effectively implemented by using Gaussian-Hermite Quadrature and this tool will be employed in the maximization of likelihood functions in this paper (Butler and Moffitt, 1982). This method is less time consuming and more efficient in comparison with the other alternative based on Monte Carlo integration with a proper simulator (Gourieroux and Monfort, 1993; Hajivassiliou and Ruud,1994). The details of the Gaussian-Hermite Quadrature applied in the paper is provided in the Appendix together with the likelihood functions used in the estimation process.

\section{Monte Carlo Experiments}

Various designs of $M C E$ are carried out with balanced and unbalanced panel data sets to analyze the finite sample performance of the Wooldridge method in comparison with; $i$ ) the ideal case, known initial values; ii) the worst case, exogenous initial values assumption; and iii) Heckman's reduced-form approximation. Our MCE (as all calculations in this paper) is designed in Compaq Visual Fortran, and the op-

$\overline{4}$ Note that we present the auxiliary conditional distribution of $\alpha_{i}$ with a constant $\xi_{0}$. Thus, the constant in the structural equation should be dropped. 
timization for the likelihood functions is performed using $Z X M I N$, which is very fast and robust optimization routine. ${ }^{5}$

\subsection{Balanced Panel Data}

We start to experiment with various balanced panel data sets that are based on three different data generating processes of a strictly exogenous variable. The benchmark design, $M C E_{1}$, is created by using independent and identically distributed standard normal random variates,

$$
x_{i t} \sim N[0,1]
$$

It is also important to analyze the bias with non-normal explanatory variables. It is well known in the literature that normally distributed explanatory variables may tend to produce less bias compared to $M C E$ designs with non-normally distributed explanatory variables (Ruud, 1986; Honore and Kyriazidou, 2000). We therefore modify $M C E_{1}$ by changing the data generating process of the explanatory variable to one degree of freedom chi-square distributed random variable, $\chi^{2}(1)$, which has a skewed distribution. In order to reveal the comparability with the benchmark design we also standardize this random variable. The second design, $M C E_{2}$, is thus based on the following explanatory variable, ${ }^{6}$

$$
x_{i t} \sim \frac{\chi_{(1)}^{2}-1}{\sqrt{2}}
$$

$5 \quad$ The details and the routines written for $M C E$ can be provided upon request from the author.

$6 \quad$ Note that $Z=\left(\chi_{(k)}^{2}-k\right) / \sqrt{2 k}$, where $k$ is the degrees of freedom. $Z$ is the standardized $\chi^{2}$ random variable. 
The third design, $M C E_{3}$, uses an autocorrelated explanatory variable in order to analyze the solution methods with a higher degree of intra-group variation. In this design the explanatory variable is given by the following autoregressive process,

$$
x_{i t}=\rho x_{i, t-1}+\psi_{i t}
$$

where $\psi_{i t} \sim N[0,1], x_{i 1}=\psi_{i 1}$ and $\rho=0.5$.

The data generating process for the latent dependent variable which is based on a dynamic random-effects specification is given as follows:

$$
\begin{gathered}
y_{i t}^{*}=\beta_{1} x_{i t}+\gamma y_{i, t-1}+\alpha_{i}+u_{i t} \\
y_{i 1}^{*}=\frac{\beta_{1} x_{i 1}}{1-\gamma}+\frac{\alpha_{i}}{1-\gamma}+\frac{u_{i 1}}{\sqrt{1-\gamma^{2}}}
\end{gathered}
$$

Where $i=1, \ldots, N$ and $t=2, \ldots, T ; x_{i t}$ is one of the exogenous variables given in (11), (12) or $(13) ; \alpha_{i} \sim \operatorname{iidN}\left[0, \sigma_{\alpha}^{2}\right]$; and $u_{i t} \sim i i d N\left[0, \sigma_{u}^{2}\right]$. The design adopted for the initial values in (15) aims first at including correlation between initial values and unobserved individual-effects, and second aims at creating mean stationarity in the stochastic process. The data generating process leading to a dynamic randomeffects probit model is $y_{i t}=\mathbf{1}\left(y_{i t}^{*}>0\right)$ and $y_{i 1}=\mathbf{1}\left(y_{i 1}^{*}>0\right)$; and for the dynamic random-effects tobit model is $y_{i t}=\max \left(0, y_{i t}^{*}\right)$ and $y_{i 1}=\max \left(0, y_{i 1}^{*}\right)$. The primary parameters of interest are the true state dependence, $\gamma$, and the the variance of the unobserved individual-effects, $\sigma_{\alpha}^{2}$.

True values of the parameters are set to $\beta_{1}=1, \gamma=0.5, \sigma_{\alpha}^{2}=1$ and $\sigma_{u}^{2}=1$. In each case, $L=200$ different conditioning data sets are produced for $N=200$ 
individuals who are observed $T=3,4,5,8,15$ and 20 periods. The number of quadrature points for Gauss-Hermite procedure is $30 .^{7}$ The average number of observations that are censored (for both tobit and probit cases) constitute almost 40 to $60 \%$ of the total number of observations.

The results of $M C E_{1}, M C E_{2}$ and $M C E_{3}$ are summarized in Table 1, 2 and 3, respectively. For brevity, and considering the limited space, we do not report all results obtained in the estimation process. It should be stressed in this point that the parameters which were not reported here $\left(\beta_{1}\right.$ and $\left.\sigma_{u}^{2}\right)$ were estimated with no bias almost in every case. Thus, we only present the primary parameters of interest, $\widehat{\gamma}$ and $\widehat{\sigma}_{\alpha}^{2}{ }^{8}$ Tables report mean bias and root mean square error (RMSE). Since the estimators of the type considered here often do not have finite theoretical moments, median bias and median absolute error $(M A E)$ are also presented. A negative sign on both mean and median bias shows an underestimation and positive sign shows an overestimation relative to the true values of the parameters.

Table 1 about here

The first block of Table 1, 2 and 3 presents results for the ideal case in which the initial values are nonstochastic (known initial values). Here, the sample data and the stochastic process start together at $t=1$, and all initial values are censored as $y_{i 1}=0$. This is the ideal case in which there is no initial values problem as there is no

$7 \quad$ The models are estimated for different number of quadrature points in order to check the stability of the estimated parameters. We observed that 30 quadrature points produce very stabile results.

8 The estimation results for these parameters and also the nuisance parameters which are estimated in the Heckman's reduced-form approximation and the Wooldridge method can be provided upon request from the author. 
correlation between initial values and unobserved individual-effects. Therefore, we do not expect any positive or negative bias. In line with the expectations, almost no bias is observed both for the true state dependence and the variance of unobserved individual-effects. The mean and median bias are very close to each other implying that the bias has a symmetric distribution with a decreasing variance as the duration of the panel data set is increased.

In order to create an initial values problem we operate the system $S=25$ periods before the sample data are observed for $T=3,4,5,8,15$ and 20 . This means that initial sample values (i.e. $S=26$ ) have been created with the evolution of the relationship between unobserved individual-effects and the explanatory variable during past 25 periods. The second block in Table 1,2 and 3 presents the results for the worst scenario which is based on the exogenous initial values assumption. In our case, this assumption is clearly wrong and also very strong, and thus we expect a sizeable bias on the estimated parameters. The assumption leads to a serious bias for both parameters (see footnote 7 ). The true state dependence, $\gamma$, is seriously overestimated while the variance of the effect, $\sigma_{\alpha}^{2}$, is seriously underestimated. The bias is 30 to $60 \%$ when $T=3$, but it is gradually getting smaller as the duration of the panel is increased, and there is almost no bias when the panel is longer than 15 periods. It is also observed that the result obtained in $M C E_{1}, M C E_{2}$ and $M C E_{3}$ are very close to each other. The methods have not produced bigger bias for nonnormal and autocorrelated explanatory variables and even the bias is smaller for some cases.

Third blocks in the tables present the results for the Heckman's reduced-form 
approximation. This method performs very well for all durations of the panels. The bias is very small even with the smallest duration $(T=3)$ one can use with dynamic panel data models (the bias is about 0 to $7 \%$ ). The results for the Wooldridge method are presented in the last block of the each table. This method performs almost equally well with the Heckman's reduced-form approximation. The main difference between these two methods occurs for the panels of very short durations ( $T=3$ and 4 ). Similar to the exogenous initial values assumption, the Wooldridge method also tends to produce overestimated true state dependence and underestimated variance of the unobserved individual-effects for the short panels but the bias is much smaller (15 to $25 \%$ ). For a duration which is longer than $T=5$, the magnitude of the bias produced by the Wooldridge method tends to be equal to Heckman's reduced-form approximation method. Overall and very intuitively, all methods tend to perform equally well for the panels of very long durations (longer than $T=15)$.

Table 2 about here

Table 3 about here

\subsection{Unbalanced Panel Data}

Many of the microeconomic panel data sets that are encountered in practice contain hundreds of individuals who are entering or exiting the data set in different periods leading to the panel data sets to be unbalanced. The initial values problem may 
behave different in these type of panel data sets depending on the degree of unbalancedness. Here, we will analyze the finite sample performance of the Wooldridge method using various unbalanced panel data sets by controlling for the degree of unbalancedness of the panel.

The degree of unbalancedness of panel is controlled for using the Ahrens and Pincus index (Ahrens and Pincus, 1981, Baltagi and Chang, 1994) which is defined as,

$$
r=\frac{N}{\bar{T} \sum_{i=1}^{N}\left(\frac{1}{T_{i}}\right)}
$$

where, $r$ is the degree of unbalancedness $(0<r<1)$. When it is close to 1 , the data set gets closer to a balanced panel data set, and it would be strongly balanced for $r=1$. We use different $r$ by controlling for the number of individuals $N=200$; and average number of periods $\bar{T}$ in the panel data set. Two different types of individuals are assumed with the same number of observations $N_{1}=100$ and $N_{2}=100\left(N=N_{1}+N_{2}\right)$, and with different number of time periods $T_{1}$ and $T_{2}$. The same design given with $M C E_{3}$ is used as a data generating process of the exogenous variable. The dynamic system in (14-15) is driven 25 periods before the samples are observed. The unbalanced panels are created by using average duration of $\bar{T}=$ 10 and different combinations of the durations leading $r=(1.00,0.99,0.94,0.91$, $0.84,0.75,0.64,0.51)$. For instance $r=0.51$ corresponds to severe unbalancedness with $T_{1}=3$ and $T_{2}=17$.

Table 4 and 5 present the results for dynamic random-effects probit and tobit models respectively. The tables report the results only for the exogenous initial values assumption and for the Wooldridge method. Heckman's reduced form ap- 
proximation is not affected by the unbalancedness of panel data and we do not report the results here. ${ }^{9}$ The bias and variation is increased by the degree of unbalancedness for the case of exogenous initial values assumption on both true state dependence and the variance of the individual-effects. However, the bias produced by the Wooldridge method is affected very slightly and this method tends to produce less bias even for very extreme cases. We also observe that this behaviour is the same for both probit and tobit models.

Table 4 about here

Table 5 about here

\section{Two empirical Applications}

The data used in practice includes more complicated structures. It is crucial to analyze the performance of the Wooldridge method with real microeconomic panel data sets and compare the results with the ones obtained with $M C E$. In this section, we will present two empirical applications aiming to analyze the intertemporal labour supply behavior of married woman either for labour force participation or hours of work decisions. We will illustrate the performance of the Wooldridge method for the identification of the different sources of state dependence in comparison with exogenous initial values assumption and Heckman's reduced-form approximation as in the case of $M C E$.

$\overline{9}$ However, they can be reported upon request from the author. 
The dynamics of labour supply behavior of women has been one of the important issues in labour economics. In our empirical applications, we first focus on the relationships between labour force participation decisions, fertility decisions and non-labor income of married women in Sweden using a dynamic random-effects probit model. Second, the hours of work decisions of the same individuals will be analyzed using a dynamic random-effects tobit model. In both applications, we use husbands' labour income as a proxy for non-labour income for married women. As in the studies of Heckman (1978) and Hyslop (1999), we try to distinguish different sources of state dependence. A true or structural state dependence on labour supply behavior caused by the past participation experiences and a spurious state dependence due to persistent unobserved individual-effects which can alter participation propensities independently from actual participation experiences. Controlling for different sources for persistence can be rationalized by past experiences that can be perceived by employers as signal for low productivity, time out for skills or search cost which differ across participation states.

The data that we use is a randomly sampled portion of registered data of Sweden (LINDA). ${ }^{10}$ Our sample selection criteria is continuously married $N=2000$ couples, aged $20-60$ in 1992 and followed until 2001 (10 periods), with positive husband's annual earnings and hours worked each year. Table 6 presents the characteristics of the sample used in our empirical applications below.

\section{Table 6 about here}

$\overline{10}$ Features of LINDA can be found in Edin and Fredriksson (2000) 


\subsection{Intertemporal participation decisions of Swedish woman,}

1992-2001

We use a very similar model that is used by Hyslop (1999). Individuals current participation decision depends on their previous employment status, fertility variables, non-labour income and unobserved individual-effects. The specification of the model estimated here is given in (1) and (2). The dependent variable is an indicator variable which is 1 for an individual $i(i=1, \ldots, 2000)$ and period $t(t=2, \ldots, 10)$, if she is employed and 0 otherwise. The unobserved individual-effects assumed as correlated with observed individual characteristics. The error-terms assumed as $u_{i t} \sim \operatorname{iidN}[0,1]$ for identification. The actual disturbance process is assumed as serially uncorrelated and the serial correlation is assumed as constant and equal to the proportion of the variance explained by the unobserved individual-effects. The exogenous variables which are included are age, age-squared, place of birth, highest level of education, fertility variables and non-labour income. Place of birth is an indicator variable and equal to 1 if the individual was born in Sweden and 0 otherwise. We use three indicator variables for to control for education: primary $=1$ (Grundskola degree, 9 years of education), secondary $=1$ (Gymnasium (high school) degree, more than 9 years but less than 12 years of education), university $=1$ (education more than Gymnasium). Similar to Hyslop (1999), the fertility variables are considered as number of children aged $0-2\left(\# K i d s_{i t}(0-2)\right), 3-5\left(\# K i d s_{i t}(3-5)\right)$ and $6-17$ $\left(\# K i d s_{i t}(6-17)\right)$. The non-labour income is separated as permanent and transitory effects. The permanent non-labour income is calculated as within means of husband's labour earnings over time periods, and the transitory non-labour income 
calculated as deviations from the within means of the husband's labour earnings.

The unobserved taste for working is possibly correlated with fertility variables and non-labour income (Hyslop, 1999). Thus, the unobserved individual-effects should be conditioned on these variables. To keep the analysis simple, and without loosing the generality, we specify the distribution based only on fertility variables using the within means of the fertility variables following the correlated randomeffects model: ${ }^{11}$

$$
\alpha_{i}=\pi_{0}+\pi_{1} \overline{\# K i d s_{(0-2)}}+\pi_{2} \overline{\# K i d s_{(3-5)}}+\pi_{3} \overline{\# K i d s_{(6-17)}}+\psi_{i}
$$

where $\psi_{i} \sim i i d N\left[0, \sigma_{\psi}^{2}\right]$. The auxiliary distribution for the unobserved individualeffects is modelled using Wooldridge method as follows:

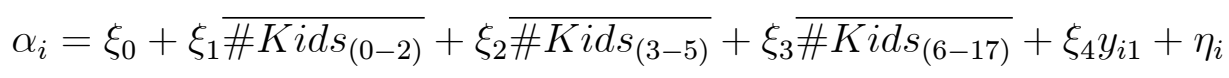

where $\eta_{i} \sim \operatorname{iidN}\left[0, \sigma_{\eta}^{2}\right]$.

Having assumed that the distribution of the unobserved individual-effect is correctly specified, we use (17) with the exogenous initial values assumption and with Heckman's method. Note that the only difference between exogenous initial values assumption and Wooldridge method is that the later includes the initial values, $y_{i 1}$ (first period employment status). Thus, by applying a correlated random-effects

$\overline{11}$ Note that the distribution of the heterogeneity is specified in Hyslop (1999) as follows

$$
\alpha_{i}=\sum_{s=0}^{T}\left(\delta_{1 s}\left(\# K i d s_{(0-2)}\right)+\delta_{2 s}\left(\# K i d s_{(3-5)}\right)+\delta_{3 s}\left(\# K i d s_{(6-17)}\right)\right)+\sum_{s=0}^{T-1} \delta_{4 s} y_{m i s}+\eta_{i}
$$

where $y_{m}$ is the transitory lon-labour income and $\eta_{i} \mid x_{i} \sim N\left[0, \sigma_{\eta}^{2}\right]$ 
model, we can also directly compare the results from these three approaches. The reduced-form specification for the Heckman's method includes a constant, the initial sample values of age, age-squared, place of birth, highest level of education and non-labour income. ${ }^{12}$

Table 7 about here

The estimation results are summarized in Table 7 by solution methods. For brevity, the table reports results only for the true state dependence and variance of unobserved individual-effects. ${ }^{13}$ In each raw, the first figure is the estimated coefficient, the second figure (in parentheses) is the standard error ${ }^{14}$ and the third figure (in brackets and bold) is the relative difference between Heckman's reducedform approximation and the other methods. A positive relative difference represents an overestimation and a negative values is an underestimation in comparison with the Heckman's method. ${ }^{15}$ Additionally, in order to see the performance of the Wooldridge method with respect to duration of the panel, we estimate the same model with different durations from $T=3$ to $T=10$. In each estimation procedure, the same individuals are used and some of the explanatory variables are

12 It is important to note that the estimation results are very much dependent on the specification of the reduced-form equation. In order to analyze the sensitivity of the result, we also tried with different specifications of the reduced-form equation using different combinations of the pre-sample information (the different combinations of the exogenous variables). It is observed that there are only minor changes on the true state dependence and the variance of the unobserved individual-effects by adding or subtracting the variables used in the reduced-form equation.

13 The estimation results of the other parameters are not included here as there are no large and systematic differences between solution methods. However, they can be provided upon request from the author.

14 The standard errors are obtained by inverting the Hessian at the maximum value of the likelihood functions.

15 Note that, here there is an intrinsic assumption that the Heckman's approximation is the true method to solve the initial values problem and the performance of the other methods has given relative to Heckman's approximation. However, it does not mean that Heckman's method is actually the true method in the practice. 
recalculated according to the duration. We also present results from pooled dynamic probit model (no unobserved individual-effects) as a base result. It is useful since it is not affected by a misspecification of unobserved individual-effects.

The results produced with real data are in line with our $M C E$. The exogenous initial values assumption produced very large true state dependence and very low variance of unobserved individual-effects for small samples. For instance, when $T=$ 3 , the relative difference between Heckman's method is 1.31 which means that the exogenous initial values assumption overestimates the true state dependence almost $131 \%$ more relative to Heckman's method. The relative difference for the variance of the unobserved individual-effects is much larger. The variance of unobserved individual-effects obtained with the Heckman's method is five times larger than that of the exogenous initial values assumption for $T=3$. When the duration is increased the difference decreases rapidly which is also in line with $M C E$. For instance, the true state dependence is overestimated only $4 \%$ and the variance is underestimated almost $11 \%$ for $T=10$. The exogenous initial values assumption produces very similar results with the pooled dynamic probit model when the duration is very small. It is may be the case that the unobserved heterogeneity is underrepresented in the short panel data sets compared to a longer one with the same individuals. It is also observed for the long panels that the pooled dynamic probit model produces increasingly larger persistence which is due to the lagged dependent variable. The reason is that this model is not able to account for the persistence in the participation sequences which is due to unobserved individual-effects.

The real data performance of the Wooldridge method, in comparison with the 
Heckman's method, is almost the same as is in $M C E$. The Wooldridge method is also not able to solve the problem of overestimation of the true state dependence and underestimation of the variance for panels of short durations. However, the relative difference between Heckman's method is much smaller compared to the relative difference between exogenous initial values assumption and Heckman's method. For $T=3$, relative difference is almost $12 \%$ and $22 \%$ for the true state dependence and variance respectively. The Wooldridge method is more successful for the true state dependence relative to variance of the unobserved individual-effects. Similar to what is found by $M C E$, the Wooldridge method performs as well as Heckman's method when the duration is larger than $T=5$. All methods tend to produce similar results for the panels of long durations.

\subsection{Dynamics of the hours of work decisions of Swedish mar- ried women,1992-2001}

The second application concentrates on hours of work decisions of married women in Sweden. Similar to the probit case, we specifically test whether there is significant true state dependence on the labour supply decisions after controlling for observed and unobserved individual characteristics. The only difference to the first empirical application is the dependent variable. Here the dependent variable is the hours of work decisions instead of an indicator variable representing the participation decisions to the labour force. The hours of work data contain either a positive value for women who are working or a zero for women who are not working. Therefore, the hours of work data is censored below zero leading to a tobit type I specification. 
We use the same observed characteristics as the above: age, age-squared, educational status, place of birth, permanent and transitory non-labour income. The unobserved individual-effects is specified as in (17) and (18), and the reduced-form specification for the Heckman's method is the same as in the probit case.

Table 8 summarizes the results by solution methods and duration of the panel from $T=3$ to $T=10$.

\section{Table 8 about here}

The impact of the initial values problem is almost the same as the above and this result is also in line with our $M C E$. The exogenous initial values assumption leads to serious bias. It overestimates the magnitude of the true state dependence and underestimates the variance of the unobserved individual-effects relative to the Heckman's method. We observe that the relative difference is a function of the duration of the panel data set. The Wooldridge method performs almost equally well compared to Heckman's method for the panels longer than $T=5$ (the difference is 5 and 13 per cent), and the difference is very close to zero for $T=10$

\section{Conclusions and discussions}

The Wooldridge method is based on a very simple and novel strategy as a solution for the initial values problem in nonlinear dynamic random-effects panel data models. This character of the Wooldridge method has attracted many researchers. However, nothing is known about its performance in comparison with the other alternative Heckman's reduced-form approximation. In this paper, using the dynamic 
random-effects probit and tobit (type I) models, the finite sample performance of the Wooldridge method is investigated in comparison with the ideal case in which the initial values are known constants, the worst case which emerges with the exogenous initial values assumption, and the Heckman's reduced-form approximation method which is based on complicated econometric techniques. Various designs of Monte Carlo Experiments are provided using balanced and unbalanced panel data sets. We also provided two real data applications which concentrate on intertemporal participation and hours of work decisions of married women in Sweden between 1992 and 2001.

The evidence obtained from $M C E$ and real data are in line with each other and confirmed the fact that a misspecification for the conditional distribution of initial values leads to serious bias on the magnitude of the true state dependence and the variance of unobserved individual-effects. The exogenous initial values assumption is one of the these cases and leads to serious overestimation of the true state dependence and serious underestimation of the variance of the unobserved individual-effects. However, this is a syndrome for small samples and the bias decrease gradually as the duration of the panel data set increase.

We also obtain clear evidence on the performance of the Wooldridge method in comparison with the Heckman's method. The key parameter to select one of them in the practice is mainly the duration of the panel data set. The Wooldridge method does not specify an explicit conditional probability distribution for the initial values, and the bias obtained with this method is behaviourally the same as the exogenous initial values assumption for very short panels. The persistence which is due to 
structural reasons is overestimated whereas the persistence due to unobserved timeinvariant individual characteristics is underestimated. However, the bias produced by the Wooldridge method is much smaller than the bias produced by the exogenous initial values assumption. The main message of our results is that the Wooldridge method can be used instead of Heckman's method only for the moderately long panels, but for the short panels Heckman's approximation is suggested. The other message of the paper is very intuitive. For the panels of longer durations, the relative importance of the initial period likelihood in the joint likelihood of all periods would be lower leading to a lower bias which is due to the initial values problem. This is what we observe in our $M C E$ and real data applications that performance of all methods tends to be equal for the panels of long durations.

\section{References}

[1] Ahrens, H. and Pincus, R. 1981. On Two Measures of Unbalancedness in a Oneway Model and Their Relation to Efficiency. Biometric Journal 23: 227-235.

[2] Arellano, M. and Honore, B. 2001. Panel Data Models. Some Recent Developments. Handbook of Econometrics, Vol V, Elsevier Science, Amsterdam.

[3] Arellano, M. and Bover, O. 1997. Estimating Dynamic Limited Dependent Variable Models From Panel Data. Investigaciones Economicas 21: 141-65 
[4] Arellano, M. and Carrasco, R. 2003. Binary Choice Panel Data Models with Predetermined Variables. Journal of Econometrics 115: 125-157

[5] Arellano, M. and Hahn, J. 2006. Understanding Bias in Nonlinear Panel Models: Some Recent Developments. In: R. Blundell, W. Newey, and T. Persson (eds.): Advances in Economics and Econometrics, Ninth World Congress, Cambridge University Press, forthcoming.

[6] Baltagi, B.H. and Chang, Y.J. 1995. Incomplete Panels. Journal of Econometrics 62: $67-89$.

[7] Butler, J. S. and Moffitt, R. 1982. A Computationally Efficient Quadrature Procedure for the One-Factor Multinomial Probit Model. Econometrica 50: $761-764$

[8] Blundell, R.W. and Smith, R.J. 1991. Initial Conditions and Efficient Estimation in Dynamic Panel Data Models. Annales d'Economie et de Statistique 20/21: 109-123

[9] Bulundell, R. and Bond, S. 1998. Initial conditions and Moment Conditions in Dynamic Panel Data Models. Journal of Econometrics 87: 115-143

[10] Card, D. and Sullivan, D., 1988. Measuring the Effect of Subsidized Training Programs on Movements In and Out of Employment. Econometrica 56: 497-530

[11] Contayannis, P., Jones, A. M., and Rice, N. 2004. The dynamics of health in the British Household Panel Survey, Journal of Applied Econometrics 19: 473-503 
[12] Chamberlain, G. 1984. Panel Data, in Handbook of Econometrics, Vol. II, edited by Zvi Griliches and Michael Intriligator. Amsterdam: North Holland.

[13] De Jong, R. and Herrera, A. 2005. Dynamic censored regression and the Open Market Desk reaction function. Ohio State University and Michigan State University.

[14] Edin, P.A. and Fredriksson, P. 2000. "LINDA - Longitudinal INdividual DAta for Sweden", Working Paper 2000:19, Department of Economics, Uppsala University.

[15] Heckman, J.J. 1978. "Simple statistical models for discrete panel data developed and applied to test the hypothesis of true state dependence against the hypothesis of spurious state dependence", Annales de l'INSEE 30-31: 227-269

[16] Heckman, J.J. 1981. The Incidental Parameters Problem and the Problem of Initial Conditions in Estimating a Discrete Time - Discrete Data Stochastic Process, in Structural Analysis of Discrete Panel Data with Econometric Applications, ed. by C. Manski and D. McFadden, Cambridge: MIT Press.

[17] Hajivassiliou, V. and Ruud, P. 1994. Classical Estimation Methods for LDV Models using Simulation, in R. Engle \& D. McFadden, eds. Handbook of Econometrics, Vol IV, 2384-2441

[18] Honore, B. 1993. Orthogonality-conditions for Tobit model with fixed effect and lagged dependent variables. Journal of Econometrics 59: 35-61 
[19] Honore, B. 2002. Nonlinear Models with Panel Data. Portuguese Economic Journal 1: 163-179.

[20] Honore, B. and Kryiazidou, E. 2000. Panel Data Discrete Choice Models with Lagged Dependent Vairables. Econometrica 74: 611-629

[21] Honore, B. and Tamer, E. 2006. Bounds on Parameters in Panel Dynamic Discrete Choice Models. Econometrica 68: 839-874

[22] Hsiao, C. 2003. Analysis of Panel Data. 2nd ed. Cambridge University Press, Cambridge.

[23] Hu, L. 2002. Estimation of a censored dynamic panel-data model. Econometrica 70: $2499-2517$

[24] Hyslop, D. R. 1999. State Dependence, Serial Correlation and Heterogeneity in Intertemporal Labor Force Participation of Married Women. Econometrica 67: $1255-1294$

[25] Gourieroux, C., and Monfort, A. 1993. Simulation-based Inference: A Survey with Special Reference to Panel Data Models. Journal of Econometrics 59: $5-33$

[26] Neyman, J. and Scott, E. 1948. Consistent Estimates Based on Partially Consistent Observations. Econometrica 16: 1-32

[27] Ruud, P. 1986. Consistent estimation of limited dependent variable models despite misspecification of distribution. Journal of Econometrics 32: 157-187 
[28] Wooldridge, J.M. 2005. Simple solutions to the initial conditions problem in dynamic, nonlinear panel-data models with unobserved heterogeneity. Journal of Applied Econometrics 20: 39-54

\section{Appendix}

The Gaussian-Hermite Quadrature, that we implement to calculate the integral in the likelihood function (5), is based on approximating the Gaussian integral, $\int_{-\infty}^{\infty} e^{-v^{2}} h(v) d v \approx \sum_{m=1}^{M} w_{m} h\left(v_{m}\right)$; where $v_{1}, v_{2}, \ldots, v_{M}$ roots of the Hermite polynomial $H(v) ; M(m=1,2, \ldots, M)$ is the number of evaluation points in the approximation process; and $w_{m}$ is the corresponding weight for the root $v_{m}$. The pairs of $\left(v_{m}, w_{m}\right)$, for different $M$, can be easily obtained by using existing tables in the literature. Having assumed that the unobserved individual-effects is normally distributed, and given the conditional distribution of initial values, the integral in the likelihood function of probit model can be calculated as follows:

$$
=\frac{1}{\sqrt{2 \pi} \sigma_{\alpha}} \int_{-\infty}^{\infty}\left[f_{i 1}\left(y_{i 1} \mid x_{i t}, \alpha_{i}\right) \prod_{t=2}^{T} \Phi\left\{D_{i t}\left(x_{i t}^{\prime} \beta+\gamma y_{i, t-1}+\sigma_{\alpha} \alpha_{i}\right)\right\}\right] e^{-\frac{\alpha^{2}}{\left(\sqrt{2} \sigma_{\alpha}\right)^{2}}} d \alpha
$$

where $D_{i t}=\left(2 y_{i t}-1\right)$. By using the transformation, $\widetilde{\alpha}=\frac{\alpha}{\sqrt{2} \sigma_{\alpha}}, d \alpha=\sqrt{2} \sigma_{\alpha} d \widetilde{\alpha}$ and considering that $v_{m}=\widetilde{\alpha}$,

$$
h\left(v_{m}\right)=f_{i 1}\left(y_{i 1} \mid x_{i t}, \sqrt{2} \sigma_{\alpha} v_{m}\right) \prod_{t=2}^{T} \Phi\left\{D_{i t}\left(x_{i t}^{\prime} \beta+\gamma y_{i, t-1}+\sqrt{2} \sigma_{\alpha} v_{m}\right)\right\}
$$


The full log-likelihood function is,

$$
\log \mathcal{L}=\sum_{i=1}^{N} \ln \left[\frac{1}{\sqrt{\pi}} \sum_{m=1}^{M} w_{m} h\left(v_{m}\right)\right]
$$

Note that the solution methods for the initial values can also be easily adopted to the above procedure. The exogenous initial values assumption leads to ignoring $f_{1}($.$) , and it can be taken outside of (19). The likelihood function for the Heckman's$ method is given as,

$$
h\left(v_{m}\right)=\Phi\left\{D_{i 1}\left(z_{i 1} \pi+\sqrt{2} \lambda \sigma_{\alpha} v_{m}\right)\right\} \prod_{t=2}^{T} \Phi\left\{D_{i t}\left(x_{i t}^{\prime} \beta+\gamma y_{i, t-1}+\sqrt{2} \sigma_{\alpha} v_{m}\right)\right\}
$$

and for the Wooldridge method,

$$
h\left(v_{m}\right)=\prod_{t=2}^{T} \Phi\left\{D_{i t}\left(x_{i t}^{\prime} \beta+\gamma y_{i, t-1}+\xi_{0}+\xi_{1} y_{i 1}+\xi_{2} \bar{x}_{i}+\sqrt{2} \sigma_{\eta} v_{m}\right)\right\}
$$

The same procedure can be easily implemented for the integral which will appear in the likelihood function of the dynamic random-effects tobit model by simply using the same strategy given the above. 


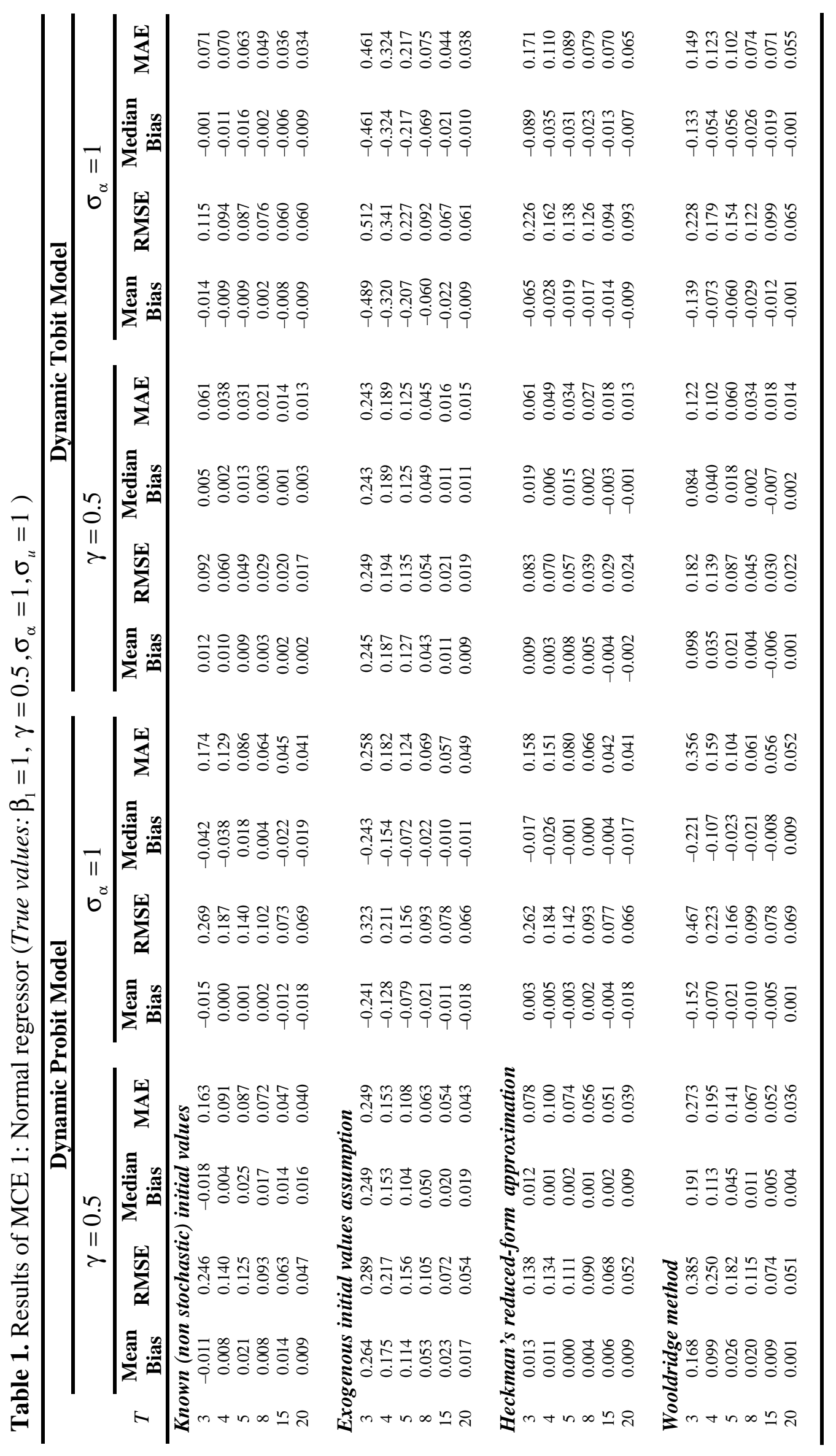




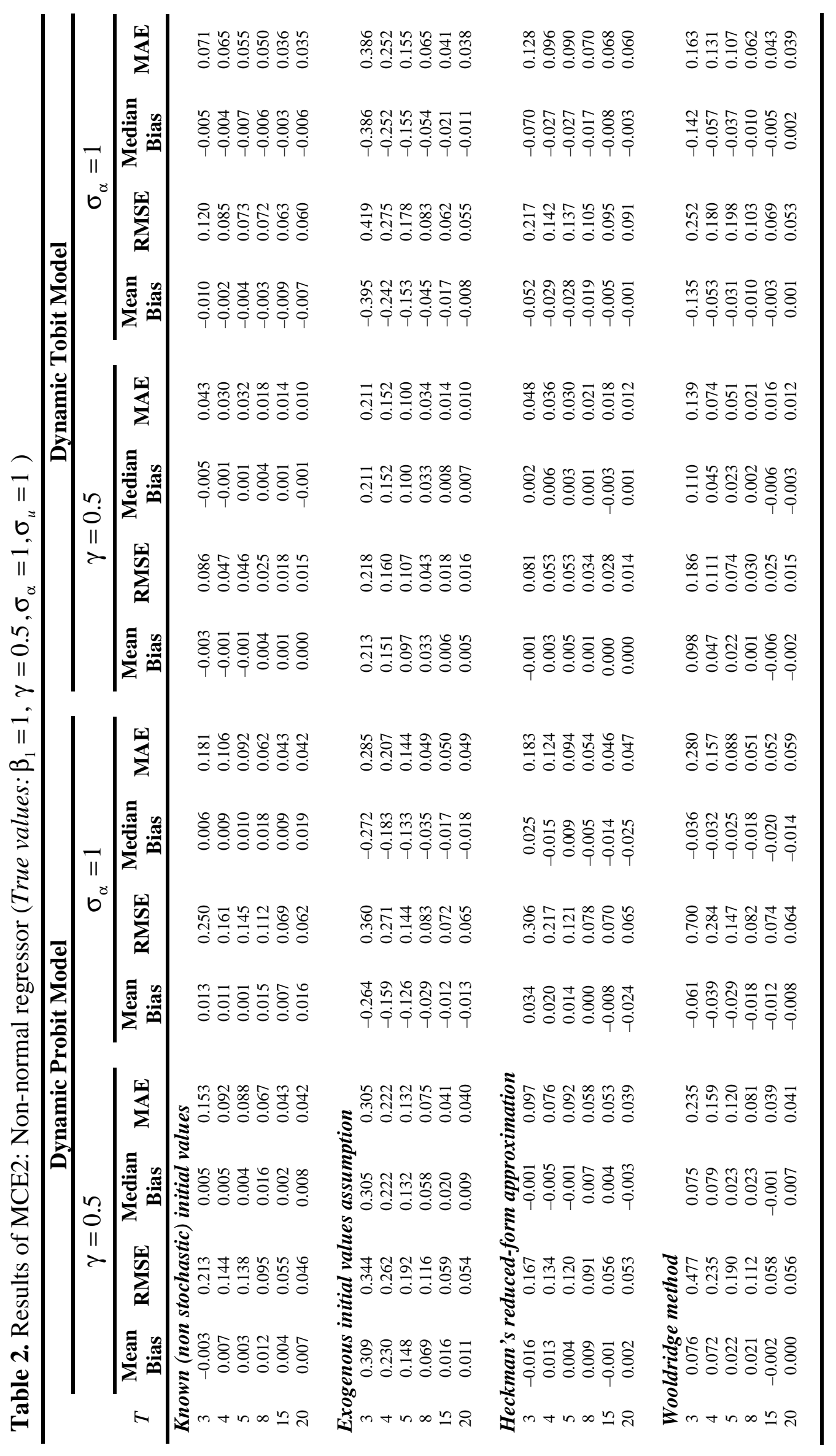




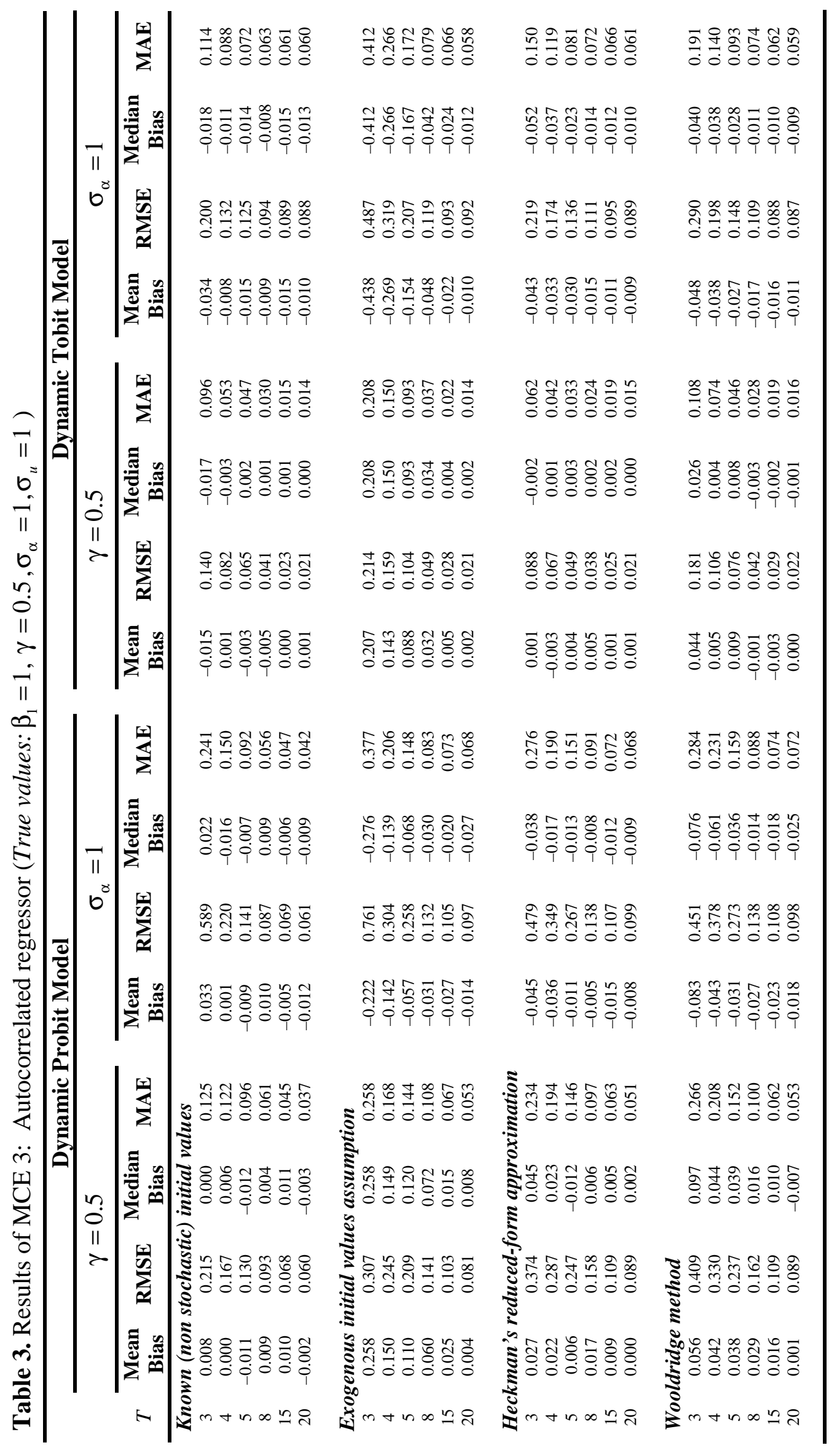




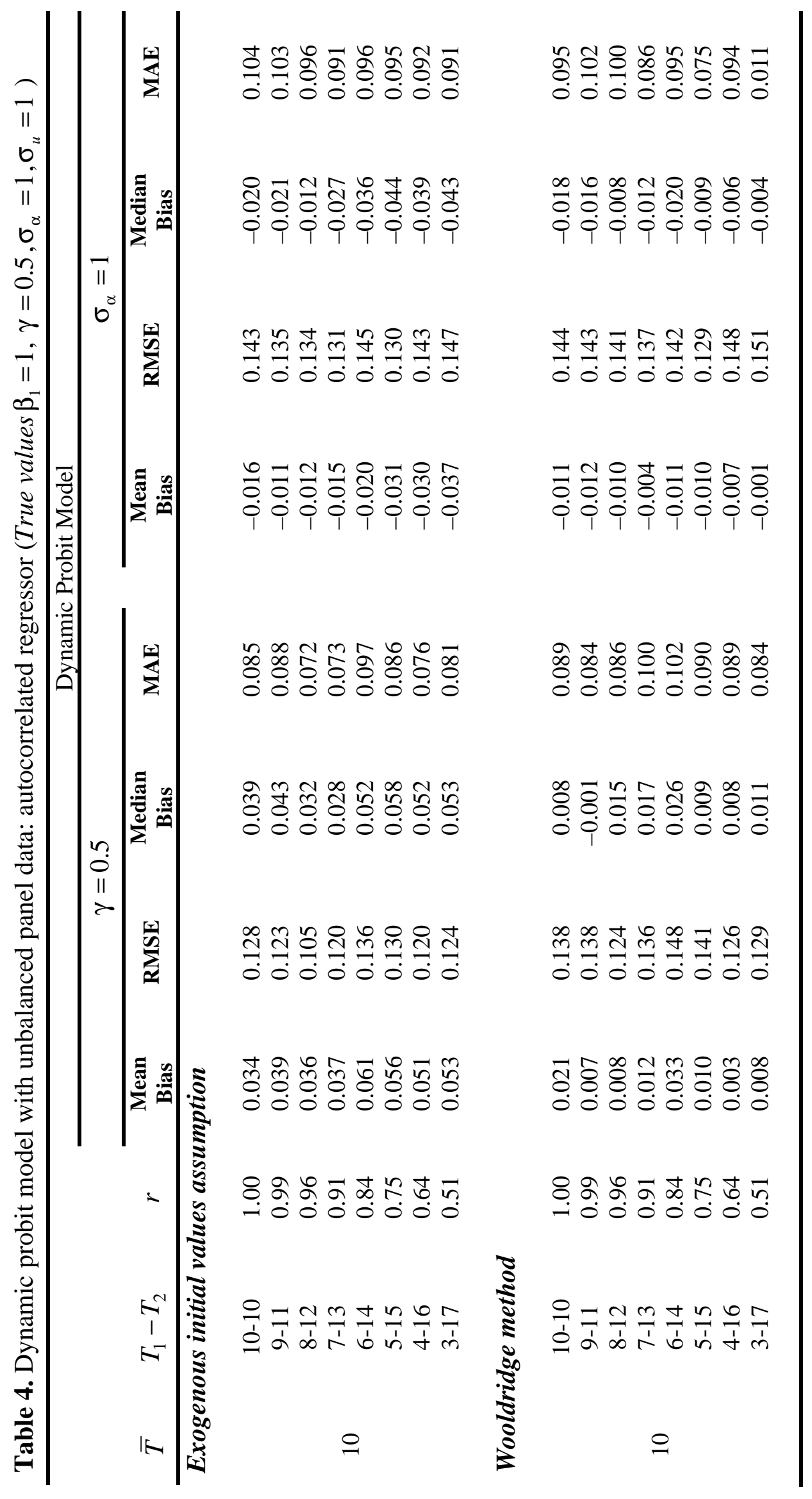




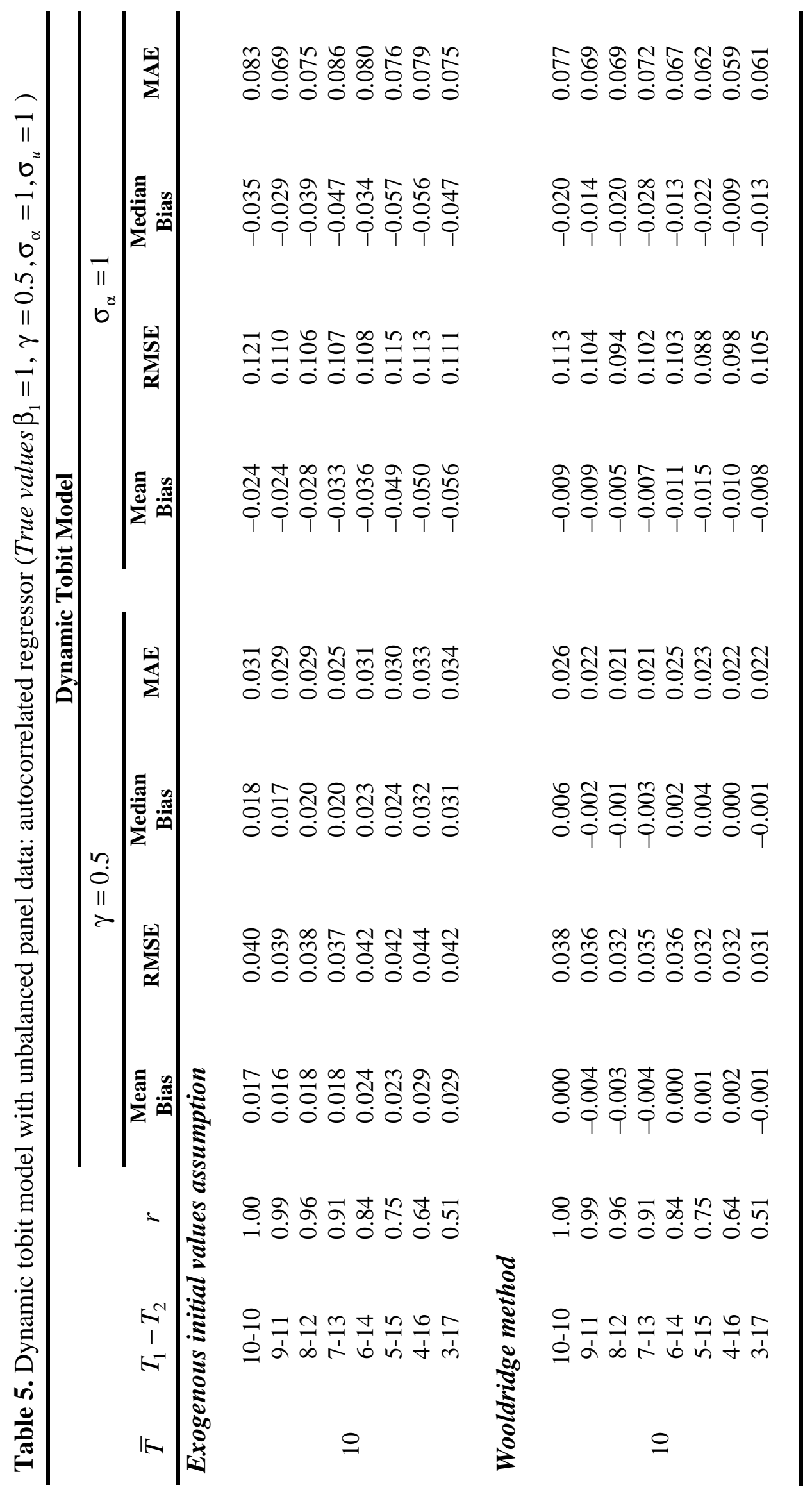




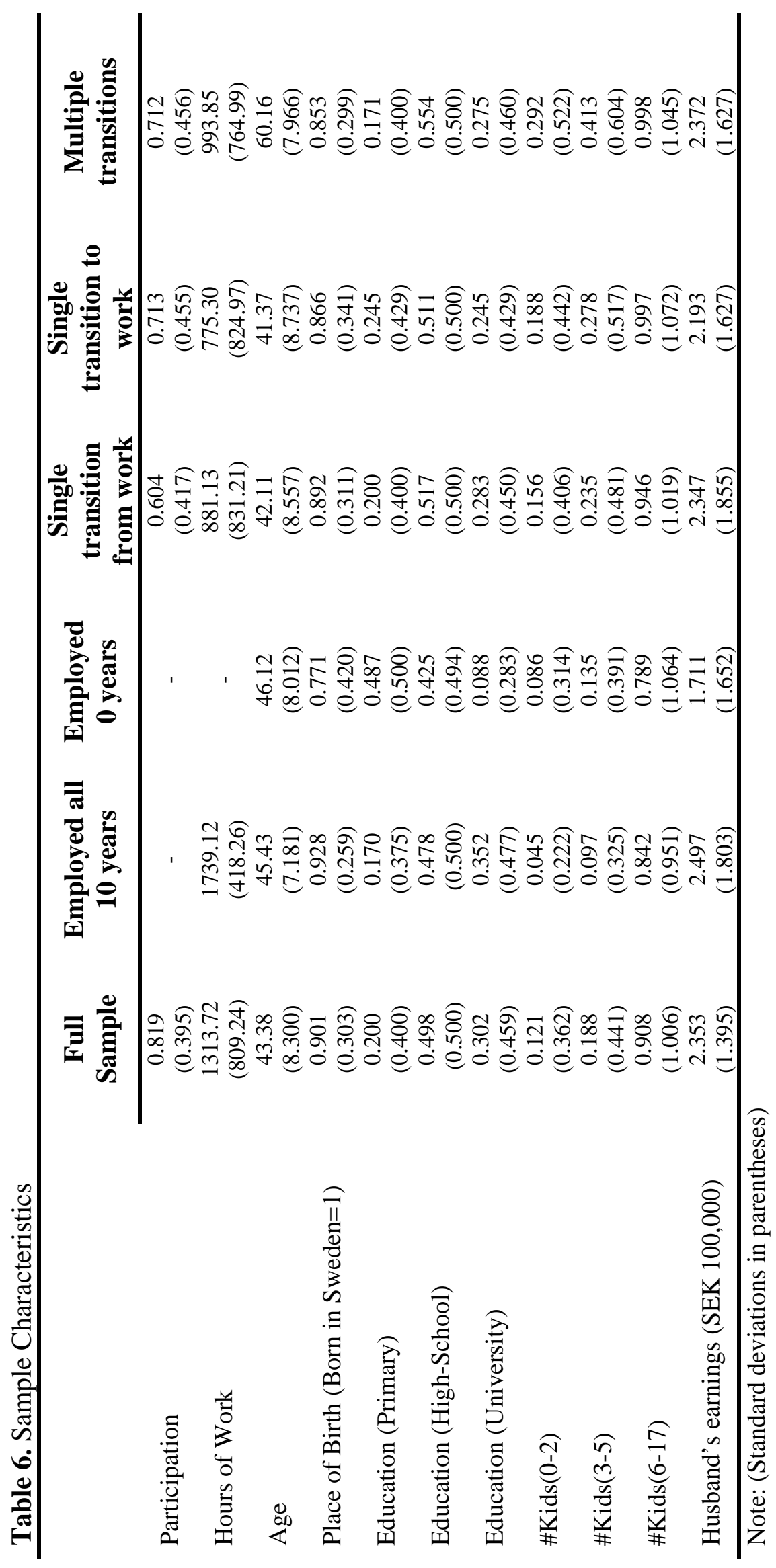




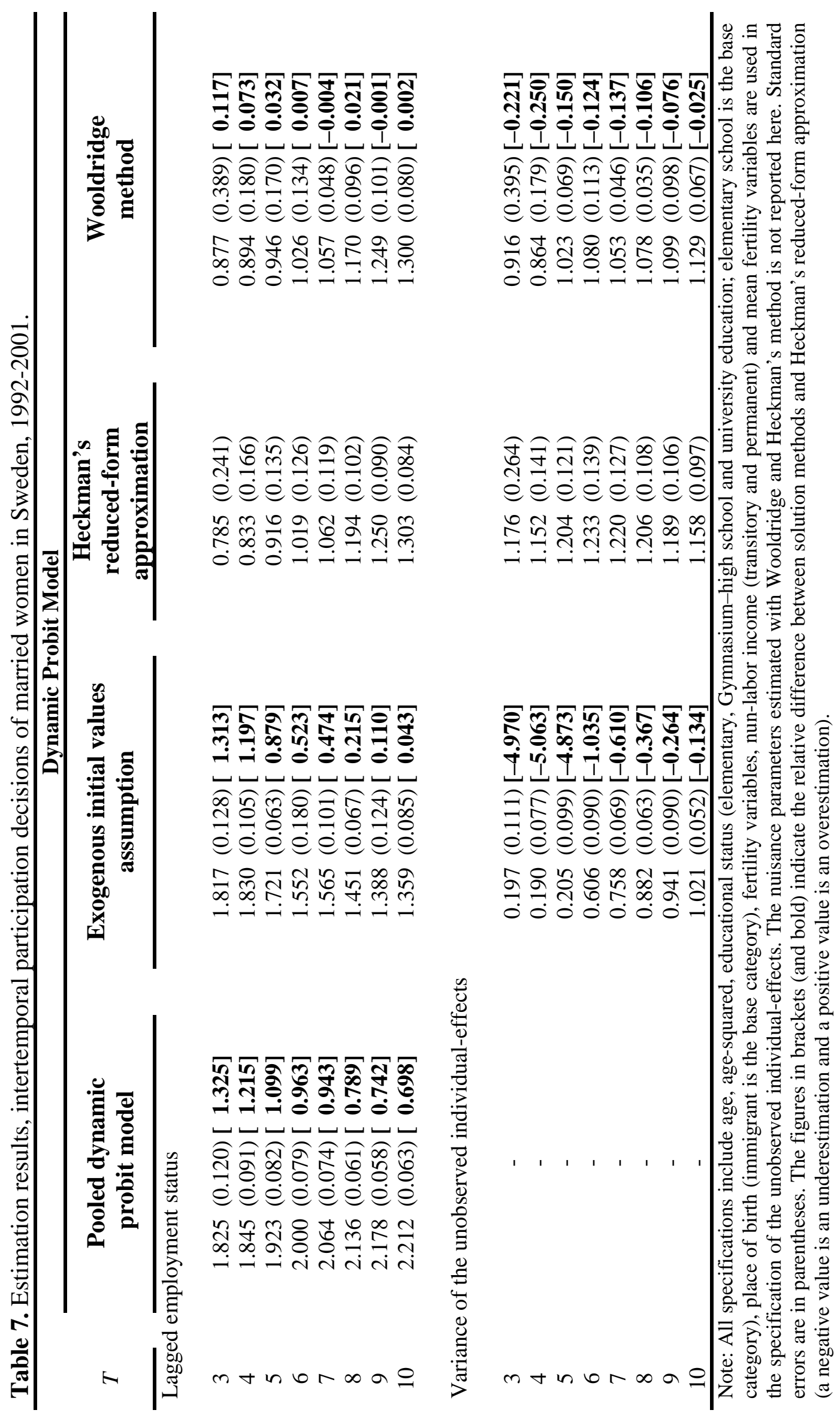




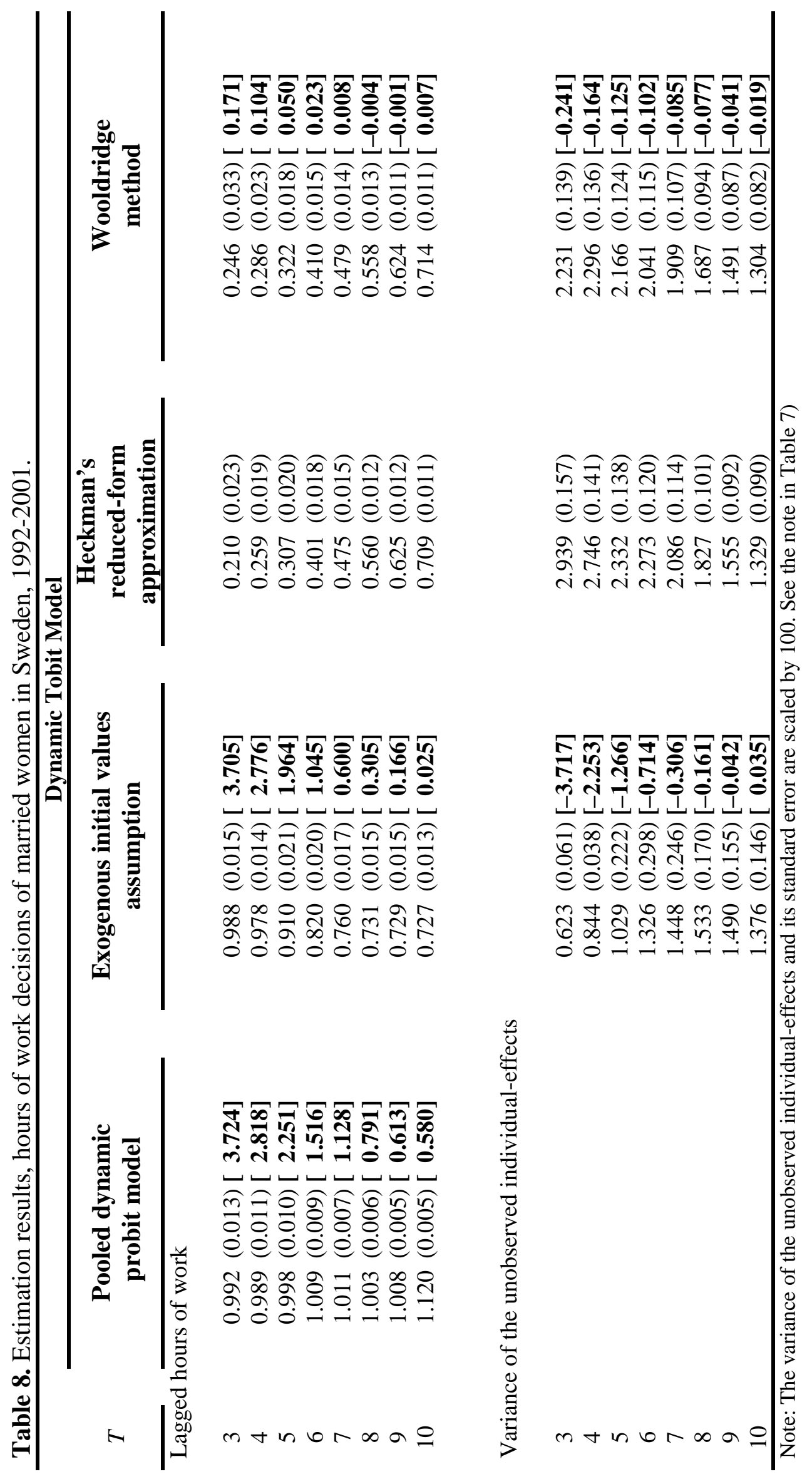

